\title{
Intransitive Permutation Groups with Bounded Movement Having Maximum Degree
}

\author{
Behname Razzaghmaneshi \\ Department of Mathematics \\ Islamic Azad University,Talesh Branch,Talesh, Iran \\ Email: behnamrazagi@yahoo.com
}

\begin{abstract}
Let $G$ be a permutation group on a set $\Omega$ with no fixed points in $\Omega$ and let $m$ be a positive integer. If for each subset $\Gamma$ of $\Omega$ the size $\left|\Gamma^{g} \backslash \Gamma\right|$ is bounded, for $g \epsilon G$, we define the movement of $g$ as the $\max \left|\Gamma^{g} \backslash \Gamma\right|$ over all subsets $\Gamma$ of $\Omega$. In this paper we classified all of permutation groups on set $\Omega$ of size $3 m+1$ with 2 orbits such that has movement $m$.
\end{abstract}

2000 AMS classification subjects: 20B25

\section{Introduction}

Let $G$ be a transitive permutation group on a set $\Omega$ such that $G$ is not 2-group and let $m$ be a positive integer. In [ ], C.E.Oraeger shown that if $\left|\Gamma^{g} \backslash \Gamma\right| \leq m$ for every subset $\Gamma$ of $\Omega$ and all $g \in G,|\Omega| \leq\left\lfloor\frac{2 m p}{p-1}\right\rfloor$, where $p$ is the least odd prime dividing $|G|$. If $p=3$ the upper bounded for $|\Omega|$ is $3 m$, and the groups $G$ attaining this bound where classified in the work of Gardiner([2]), Mann and the C.E.Praeger ([3]). Here we show that if $G$ be a intrasitve permutation group on set $\Omega$ of size $3 m+1$ with 2 orbits such that has movement $m$, and let $B$ is the semi-direct product of $Z_{2}^{2} \cdot Z_{3}$. Then $G$ is satisfy one of the following : $G_{1}=B \times H^{d}$ or $G_{2}=A_{4} \times H^{d}$, where $H=Z_{3}$ or $S_{3}, d=m-2$, and $A_{4}$ is the permutation group on 4 elements. Let $G$ be a permutation group on a set $\Omega$ with no fixed points in $\Omega$ and let $m$ be a positive integer. If for a subset $\Gamma$ of $\Omega$ the size $\left|\Gamma^{g} \backslash \Gamma\right|$ is 
bounded, for $g \in G$, we define the movement of $\Gamma$ as $\operatorname{move}(\Gamma)=\max _{g \in G}\left|\Gamma^{g} \backslash \Gamma\right|$. If move $(\Gamma) \leq m$ for all $\Gamma \subseteq \Omega$,then $G$ is said to have bounded movement and the movement of $G$ is define as the maximum of move $(\Gamma)$ over all subsets $\Gamma$, that is,

$$
m:=\operatorname{move}(G):=\sup \left\{\left|\Gamma^{g} \backslash \Gamma\right| \mid \Gamma \subseteq \Omega, g \in G\right\} .
$$

This notion was introduced in [3]. By [3,Theorem 1], if $G$ has bounded movement $m$,then $\Omega$ is finite. Moreover both the number of $G$-orbits in $\Omega$ and the length of each $G$-orbit are bounded above by linear functions of $m$.In particular it was shown that the number of $G$-orbits is at most $2 m-1$. 1.The main result is the following theorem.

Theorem 1.1. Let $G$ a permutation group on set $\Omega$ of size $3 m+1$ with 2 orbits such that has movement $m$, and let $B$ is the semi-direct product of $Z_{2}^{2} . Z_{3}$. Then $G$ is $G_{1}=B \times H^{d}$ or $G_{2}=A_{4} \times H^{d}$, where $H=Z_{3}$ or $S_{3}, d=m-2$, and $A_{4}$ is the permutation group on 4 elements.

Note that an orbit of a permutation group is non trivial if its length is greater than 1. The groups described below are examples of permutation groups with bounded movement equal to $m$ which have exactly $\frac{1}{2}(3 m-1)+\frac{1}{p}$ nontrivial orbits.

\section{Examples and Preliminaries}

Let $1 \neq g \in G$ and suppose that $g$ in its disjoint cycle representations has $t$ nontrivial cycles of lengths $l_{1}, \ldots, l_{t}$, say. We might represent $g$ as

$g=\left(a_{1} a_{2} \ldots a_{l_{1}}\right)\left(b_{1} b_{2} \ldots b_{l_{2}}\right) \ldots\left(z_{1} z_{2} \ldots z_{l_{t}}\right)$. Let $\Gamma(g)$ denote a subset of $\Omega$ consisting $\left\lfloor l_{i} / 2\right\rfloor$ points from the $i$ th cycle, for each $\mathrm{i}$, chosen in such a way that $\Gamma(g)^{g} \cap \Gamma(g)$ $=\varnothing$. For example, we could choose

$\Gamma(g)=\left\{a_{2}, a_{4}, \ldots, a_{k_{1}}, b_{2}, b_{4}, \ldots, b_{k_{2}}, \ldots, z_{2}, z_{4}, \ldots, z_{k_{t}}\right\}$, where $k_{i}=l_{i}-1$ if $l_{i}$ is odd and $k_{i}=l_{i}$ if $l_{i}$ is even. Note that $\Gamma(g)$ is not uniquency determined as it depends on the way each cycle is written . For any set $\Gamma(g)$ consists of every point of very cycle of $g$. From the definition of $\Gamma(g)$ we see that

$$
\left|\Gamma(g)^{g} \backslash \Gamma(g)\right|=|\Gamma(g)|=\sum_{i=1}^{t}\left\lfloor l_{i} / 2\right\rfloor .
$$

The next lemma shows that this quantity is an upper bound for $\left|\Gamma^{g} \backslash \Gamma\right|$ for an arbitrary subset $\Gamma$ of $\Omega$.

Lemma 2.1. [5, Lemma 2.1]. Let $G$ be a permutation group on a set $\Omega$ and 
suppose that $\Gamma \subseteq \Omega$. Then for each $g \in G,\left|\Gamma^{g} \backslash \Gamma\right| \leq \sum_{i=1}^{t}\left\lfloor l_{i} / 2\right\rfloor$, where $l_{i}$ is the length of the $i$ th cycle of $g$ and $t$ is the number of nontrivial cycles of $g$ in its disjoint cycle representation. This upper bound is attained for $\Gamma=\Gamma(g)$ defined above .

Now we will show that there certainly is an infinite family of 3 -groups for which the maximum bound obtained in Theorem 1.1 holds .

Example 2.2 . Let $d$ be a positive integer $\Omega=\Omega_{1} \cup \Omega_{2}$ be a set of size 7 , such that $\Omega_{1}=\{1,2,3\}$ and $\Omega_{2}=\{1,2,3,4\}$. Moreover, suppose that $Z_{2}^{2} \cong\left\langle\left(12^{\prime}\right)\left(34^{\prime}\right),\left(1^{\prime} 3^{\prime}\right)(24)\right\rangle$ and $Z_{3} \cong\left\langle(123)\left(1^{\prime} 2^{\prime} 3^{\prime}\right)\right\rangle$. Then the semi-direct product $G=Z_{2}^{2} Z_{3}$ with normal subgroup $G=Z_{2}^{2}$ is a permutation group on a set $\Omega$ with 2 -orbits which movement 2 , since each non-identity element of $G$ has two cycle of length 2 or two cycle of length 3 .

Example 2.3 .Let $Z_{2}^{2}=\langle x\rangle$ and $Z_{3}=\langle y\rangle$, and write $G=\left\{x^{i} y^{j} z \mid z \in Z_{3}^{d}\right\}$. Note that $y$ lies in $G$. If $x$ lies in $G$, then $G=\left(Z_{3} . Z_{2}^{2}\right) \times Z_{3}$. If $x \notin G, x^{2}$ lies in $G$. We then consider a subgroup $T=\left\{z \in Z_{3}^{d} \mid z \in G\right\}$ and a subset $S=\left\{z \in Z_{3}^{d} \mid y z \in G\right\}$ of $Z_{3}^{d}$. Let $\Omega_{1}, \ldots, \Omega_{d}, d G-$ orbits and $\Delta=\bigcup_{i=1}^{d} \Omega_{i}$, $\Delta^{\prime}=\Omega \backslash \Delta$ and $K$ the pointwise stabilizer on $\Delta$. Since the permutation group induced by $G / K$ on is an elementary abelian 3-group $Z_{3}^{d}$, we have $T \cap S=$ and $T \cup S=Z_{3}^{d}$. If $z^{\prime}$ and $z^{\prime \prime}$ lie in $S$, then $y z^{\prime} y z^{\prime \prime} \in G$ and so does $z^{\prime} z^{\prime \prime} \in G$. This means $S \subset \alpha T$ for some $\alpha \in Z_{3}^{d} \backslash T$, and $Z_{3}^{d}=T \cup \alpha T$. Hence $G=\left\{x^{i} y^{3 j+1} \alpha t \mid t \in T\right\} \cup\left\{x^{i} y^{3 j} t \mid t \in T\right\}=\left\{x^{i}(y \alpha)^{j} t \mid t \in T\right\}$. Let $H=\left\{x^{i}(y \alpha)^{j}\right\}$. Then $T \cap H=\{1\}$ and $H T=G$. Since $T$ and $H$ are normal subgroups of $G$, we have $G=H \times T$. Since $H=\left\{x^{i}(y \alpha)^{j}\right\} \simeq Z_{3} . Z_{2}^{2}$ and $T \simeq Z_{3}^{(d-1)}$, we have $G \simeq\left(Z_{3} \cdot Z_{2}^{2}\right) \times Z_{3}^{d}$. This is complete the proof of Theorem 1.1.

Corrolary For every $m>2$, the theorem of this paper has answers .

\section{Proof of Theorem 1.2.}

In this section we prove Theorem 1.2, we show first that a minimal counterexample to Theorem 1.2, must be a nonabelian simple group acting primitively on $\Omega$. If a group $G$ has bounded movement equal to $m$ for convenience we shall say that $G$ satisfies $\mathrm{BM}(\mathrm{m})$.

3.1.Proposition : Suppose that Theorem 1.2, is false and let $m$ be the least integer for which Theorem 1.2 false. Further let $G$ be a counterexample to Theorem 1.2, with $|G|$ minimal. Then $G$ is a nonabelian simple group 
acting primitively on $\Omega$.

Proof : Since $G$ is a counterexample to Theorem 1.2 with $|G|$ minimal, it follows that $G$ is not a 2-group,$G$ is intransitive on $\Omega, G$ satisfies $\mathrm{BM}(\mathrm{m})$, and $|\Omega|=3 m+1$. The proof proceeds in five steps.

Let $\Omega_{1}, \ldots, \Omega_{t}$ be $t$ orbits of $G$ of lengths $n_{1}, \ldots, n_{t}$. Choose $\alpha_{i} \in \Omega$ and let $H_{i}:=G_{\alpha_{i}}$, so that $\left|G: H_{i}\right|=n_{i}$. For $g \in G$, let $\Gamma(g)=\left\{\alpha_{i} \mid \alpha_{i}^{g} \neq \alpha_{i}\right\}$ be every second point of every cycle of $g$ and let $\gamma(g):=|\Gamma(g)|$. Since $\Gamma(g) \cap \Gamma(g)^{g}=\emptyset$ it follows that $\gamma(g) \leq m$ for all $g \in G$. Let $\bar{\Omega}:=\Omega_{1} \cup \ldots \cup \Omega_{t}$, and let $\bar{G}$ and $\bar{H}_{1}, \ldots, \bar{H}_{t}$ denote the finite permutation groups on $\bar{\Omega}$ induced by $\mathrm{G}$ and $H_{1}, \ldots, H_{t}$ respectively. Then $n_{i}=\left|\bar{G}_{1}: \bar{H}_{i}\right|$.

For $g \in G$, let $\bar{g} \in \bar{G}$ denote the permutation of $\bar{\Omega}$ induced by g. Then as $\gamma\left(1_{G}\right)=0$, we have $\sum_{\bar{g} \in \bar{G}} \gamma(g)<m|\bar{G}|$.

Now, Counting the pairs $(\bar{g}, i)$ such that $\bar{g} \in \bar{G}$ and $\alpha_{i}^{g} \neq \alpha_{i}$ gives

$\sum_{\bar{g} \in \bar{G}} \gamma(g)=\sum_{i}\left|\left\{\bar{g} \in \bar{G} \mid \alpha_{i}^{g} \neq \alpha_{i}\right\}\right|=\sum_{i}\left|\left\{\bar{g} \in \bar{G} \mid g \notin H_{i}\right\}\right|=\sum_{i}\left(|\bar{G}|-\left|\bar{H}_{i}\right|\right)=|\bar{G}| \sum_{i}\left(1-\frac{1}{n_{i}}\right)$.

It follows that $\sum_{i}\left(1-\frac{1}{n_{i}}\right)<m$. Since $n_{i} \geq 3, p$ for each $i$, it follows that $\sum_{i}\left(1-\frac{1}{n_{i}}\right) \geq \frac{p-1}{p}+\frac{2}{3}(t-1)$ and hence $\frac{p-1}{p}+\frac{2}{3}(t-1)<m$, that is, $t \leq \frac{1}{2}(3 m-1)+\frac{1}{p}$.

Consequently $\mathrm{G}$ has at most $\frac{1}{2}(3 m-1)+\frac{1}{p}$ orbits in $\Omega$. Now Let $m$ be a positive integer greater than 1 . Suppose that $G \leq \operatorname{Sym}(\Omega)$ with orbits, $\Omega_{2}, \ldots, \Omega_{t}$, where $t=\frac{1}{2}(3 m-1)+\frac{1}{p}$. Suppose further that $\Gamma \subseteq \Omega$ has move $(\Gamma)=m$ and that cuts across each of the G-orbits $\Omega_{i}$. For each i set $n_{i}=\left|\Omega_{i}\right|$ and $\Gamma_{i}=\Gamma \cap \Omega_{i}$. Note that $0<\left|\Gamma_{i}\right|<n_{i}$.

Claim 3.1 If Theorem 2.3 holds for the special case in which $\left|\Gamma_{i}\right|=1$ for $i=1, \ldots, \frac{1}{2}(3 m-1)+\frac{1}{p}$, then it holds in general .

Proof :Suppose that Theorem 2.3 holds for the case where each $\left|\Gamma_{i}\right|=1$. For $i=1, \ldots, t$, define $\sum_{i}:=\left\{\Gamma_{i}^{g} \mid g \in G\right\}$, and note that $\left|\sum_{i}\right| \geq 3$ since $\Gamma$ cuts across $\Omega_{i}$. Set $\Sigma=\cup_{i \geq 1} \sum_{i}$. Then $\mathrm{G}$ induces a natural action on $\Sigma$ for which the G-orbits are $\Sigma_{1}, \ldots, \Sigma_{t}$. Let $G^{\Sigma}$ denote the permutation group induced by $\mathrm{G}$ on $\Sigma$, and let $K$ denote the kernel of this action.

We claim that the t-element subset $\Gamma_{\Sigma}=\left\{\Gamma_{1}, \ldots, \Gamma_{t}\right\} \subseteq \Sigma$ has movement equal to $\mathrm{m}$ relative to $G^{\Sigma}$, and that $\Gamma_{\Sigma}$ cuts across each $\Gamma^{\Sigma}$-orbit $\Sigma_{i}$. For 
each $g \in G,\left|\Gamma^{g}-\Gamma\right| \leq m$ and hence $\left|\Gamma_{\Sigma}^{g}-\Gamma_{\Sigma}\right| \leq m$. Thus move $\left(\Gamma_{\Sigma}\right) \leq m$. Also, Since $\left|\Sigma_{i}\right| \geq 3$ and $\Gamma_{\Sigma} \cap \Sigma_{i}$ Consists of the single element $\Gamma_{i}$ of $\Sigma_{i}$, the set $\Gamma_{\Sigma}$ cuts across each of the $\frac{1}{2}(3 m-1)+\frac{1}{p}$ orbits $\Sigma_{i}$. However, it follows that the number of $G^{\Sigma}$ - orbits is at most $\frac{1}{2}\left(3\right.$.move $\left.\left(\Gamma_{\Sigma}\right)-1\right)+\frac{1}{p}$, and hence move $\left(\Gamma_{\Sigma}\right)=m$.

Thus the hypotheses of theorem 2.3 hold for the subset $\Gamma_{\Sigma} \subseteq \Sigma$ relative to $G^{\Sigma}$, and $\Gamma_{\Sigma}$ meets each $G^{\Sigma}$-orbit in exactly one point. By our assumption it follows that $t=\frac{1}{2}\left(p 3^{r}-1\right) \frac{1}{p}=\frac{1}{2}(3 m-1)+\frac{1}{p}$ for some $r>1$, and that $G^{\Sigma}=Z_{3}^{r}$ and each $\left|\Sigma_{i}\right|=3$. Further, the subgroups $H_{i}$ of $\mathrm{G}$ fixing $\Gamma_{i}$ setwise range over the $\frac{1}{2}\left(p 3^{r}-1\right)+\frac{1}{p}$ distinct subgroups which have index 3 in $G$ and which contain $K$. In particular, for each $i, H_{i}$ is normal in $G$ and hence the $H_{i}$-orbits in $\Omega_{i}$ are blocks of imprimitivity for $G$, and their number is at most $|G: H|=3$. Since $H_{i}$ fixes $\Gamma_{i}$ setwise it follows that $\Gamma_{i}$ is an $H_{i}$-orbit and $n_{i}=3\left|\Gamma_{i}\right|$.

Let $g \in G \backslash K$. Then in its action on $\Sigma$, g moves exactly $m$ of the $\Gamma_{i}$. Since the $\Gamma_{i}$ are blocks of imprimitivity for $\mathrm{G}$, each $\Gamma_{i}^{g}$ is equal to either $\Gamma_{i}$ or $\Omega_{i}-\Gamma_{i}$. It follows that $\left|\Gamma^{g} \backslash G\right|$ is equal to the sum of the sizes of the $\mathrm{m}$ subsets $\Gamma_{i}$ moved by g. However, since move $(\Gamma)=m$, each of these $\mathrm{m}$ subsets $\Gamma_{i}$ must have size 1 . Since for each i we may choose an element $\mathrm{g}$ which moves $\Gamma_{i}$, we deduce that each of the $\Gamma_{i}$ has size 1 , and that $\mathrm{K}$ is the identify subgroup. It follows that theorem 2.3 hold for $G$. Thus the claim is proved .

From now on we may and shall assume that each $\left|\Gamma_{i}\right|=1$. Let $\Gamma_{i}=\left\{\Omega_{i}\right\}$. Further we may assume that $n_{1} \leq n_{2} \leq \ldots \leq n_{t}$. For $g \in G$ let $c(g)$ denote the number of integers I such that $\omega_{i}^{g}=\omega_{i}$. Note that since move $(\Gamma)=m$, we have $c(g)>t-m=\frac{1}{2}(3 m-1)+\frac{1}{p}-m=\frac{m-1}{2}+\frac{1}{p}$ and also $c\left(1_{G}\right)=t>\frac{m-1}{2}+\frac{1}{p}$.

Lemma 3.2. If one of the orbits of $G$ has length equal to $p$, then the rest orbits of $G$ has size 3 .

Proof : Let X denote the number of pairs (g,i) such that $g \in G$, $1 \leq i \leq t$, and $\omega_{i}^{g}=\omega_{i}$. Then $X=\sum_{g \in G} c(g)$, and by our observations, $X>|G| \cdot\left(\frac{m-1}{2}+\frac{1}{p}\right)$. On the other hand, for each $i$, the number of elements of G which fix $\omega_{i}$ is $\left|G_{\omega_{i}}\right|=\frac{|G|}{n_{i}}$, and hence $X=|G| \sum_{i=1}^{t} n_{i}^{-1}$ If all the $n_{i} \geq 3$, and one of $n_{i}$ is equal to $p$, then $X \leq|G| \cdot\left(\frac{1}{p}+\frac{t-1}{3}\right)=|G|\left(\frac{1}{p}+\frac{3 m-1}{6}+\frac{1}{3 p}+\frac{1}{3}\right) \leq$ 
$|G| \cdot\left(\frac{m-1}{2}+\frac{1}{p}\right)$ (since $m \geq 3$ ) which is a contradiction. Hence $\mathrm{n}=3$.

A similar argument to this enables us to show that except one of $n_{i}$ the rest of $n_{i}$ is $n_{i}=3$, and hence that $G$ is an $3-$ group.

Lemma 3.3. The group $G=Z_{p} . Z_{3}^{r}$ for some $r \geq 2$. Moreover for each $n_{i}=3$, except one, the stabilizers $G_{\omega_{i}}(2 \leq i \leq t)$ are pair wise distinct subgroups of index 3 in $G$, and for each $g \neq 1, c(g)=\left(\frac{m-1}{2}+\frac{1}{p}\right)$.

Proof: By Lemma 3.2, except one of $n_{i}$ the rest of $n_{i}$ is $n_{i}=3$. Thus $H:=G_{\omega_{i}}$ is a subgroup of index 3 . This time we compute the number $Y$ of pairs $(g, i)$ such that $g \in G \backslash H, 2 \leq i \leq t$, and $\omega_{i}^{g}=\omega_{i}$. For each such $g, \omega_{1}^{g} \neq \omega_{1}$ and hence there are $c(g)$ of these pairs with first entry $g$. Thus $Y=\sum_{g \in G \backslash H} c(g) \geq|G \backslash H|\left(\frac{3(m-1}{2}+\frac{3}{p}\right)=|G|\left(\frac{m-1}{2}+\frac{1}{p}\right)$.

On the other hand, for each $i \geq 2$, the number of elements of $G$, which fix $\omega_{i}$ is $\left|G_{\omega_{i}} \backslash H\right|$. If $H=G_{\omega_{i}}$ then $\left|G_{\omega_{i}} \backslash H\right|=0$, while if $G_{\omega_{i}} \neq H$, then $\left|G_{\omega_{i}} \backslash H\right|=\frac{\left|G_{\omega_{i}}\right|}{3}=\frac{|G|}{3 n_{i}} \leq \frac{|G|}{9}$. Hence

$$
\begin{aligned}
& \left.Y=\sum_{i=2}^{t}\left|G_{\omega_{i}} \backslash H\right| \leq\right] \frac{|G|}{3} \sum_{i=2}^{t} \frac{1}{n_{i}} \leq \frac{|G|}{3}\left(\frac{1}{p}+\frac{t-1}{3}\right) \\
& =\frac{|G|}{3}\left(\frac{3+p(t-1)}{3 p}\right)<|G|\left(\frac{m-1}{2}+\frac{1}{p}\right)
\end{aligned}
$$

It follows that equality holds in both of the displayed approximations for $Y$. This means in particular that each $n_{i}=2$, Whence $G=Z_{p} \cdot Z_{3}^{r}$ for some $r$. Further, for each $i \geq 3, G_{\omega_{i}} \neq H$ and so $r \geq 2$. Arguing in the same way with $\mathrm{H}$ replaced by $G_{\omega_{i}}$, for some $i \geq 2$, we see that $G_{\omega_{i}} \neq G_{\omega_{j}}$ if $j \neq i$, and also if $g \in G_{\omega_{i}}$ then $c(g)=\left(\frac{m-1}{2}+\frac{1}{p}\right)$. Thus the stabilizers $G_{\omega_{i}}(1 \leq i \leq t)$ are pairwise distinct, and if $g \leq 1$ then $c(g)=\left(\frac{m-1}{2}+\frac{1}{p}\right)$. Finally we determine $m$.

Lemma 3.4.. $m=3^{r-2}$

Proof: We use the information in lemma3.3 to determine precise the quantity $X=\sum_{g \in G} c(g): X=t+(|G|-1) \cdot\left(\frac{m-1}{2}+\frac{1}{p}\right)=\frac{1}{2}(3 m-1)+\frac{1}{p}+\left(p \cdot 3^{r-1}-\right.$ $1)\left(\frac{m-1}{2}+\frac{1}{p}\right)$. On the other hand, from the proof of lemma 2.1 ,

$$
X=|G| \sum_{i=1}^{t} n_{i}^{-1}=|G| \cdot\left(\frac{1}{p}+\frac{t-1)}{3}\right)=p \cdot 3^{r-1} \cdot\left(\frac{1}{p}+\frac{3 m-1}{6}+\frac{1}{3 p}-\frac{1}{3}\right) .
$$

Thus implies that $m=3^{r-2}$.

The proof of theorem 2.3 now follows from lemmas 3.2-3.4. 


\section{References}

[1] C.E.Praeger,On permutation groups with bounded movement, J.Algebra ,144(1991),436-442.

[2] C.E.Praeger, The separation theorem for group actions, in "ordered Groups and Infinite Groups"(W.charles Holland, Ed.), Kluwer Academic, Dordrecht/Boston/Lond, 1995.

[3] A.Hassani,M.Khayaty,E.I.Khukhro and C.E.Praeger, Transitive permutation groups with bounded movement having maximum degree.J. Algebra,214(1999),317-337.

[4] A.Gardiner,C.E.Praeger,Transitive permutation groups with bounded movement,J.Algebra 168(1994)798-803.

[5] A.Mann,C.E.Praeger,ransitive permutation groups of minimal movement,J.Algebra 181(1996)903-911.

[6] H.Wielandt,Finite Permutation Groups,Academic Press,NewYork,1968.

[7] P.S.Kim, Y.Kim,Certain groups with bounded movement having the maximal number of orbits,J.Algebra 252(2002)74-83.

[8] L.Brailovsky, Structure of quasi-invariant sets, Arch.Math.,59 (1992),322326.

[9] L.Brailovsky, D.Pasechnix , C.E.Praeger, Subsets close to invarianr subset of quasi-invariant subsets for group actions ,Proc.Amer. Math.Soc. ,123(1995),2283-2295.

[10] J.R.Cho, P.S.Kim, and C.E.Praeger, The maximal number of orbits of a permutation Group with Bounded Movement, J.Algebra,214 (1999),625630 .

[11] P.M.Neumann, The structure of finitary Permutation groups, Arch. Math. (Basel) 27(1976),3-17.

[12] B.H.Neumann, Groups covered by permutable subsets, J. London Math soc., 29(1954), 236-248. 
[13] P.M.Neumann, C.E.Praeger, On the Movement of permutation Group, J.Algebra, 214, (1999)631-635. 